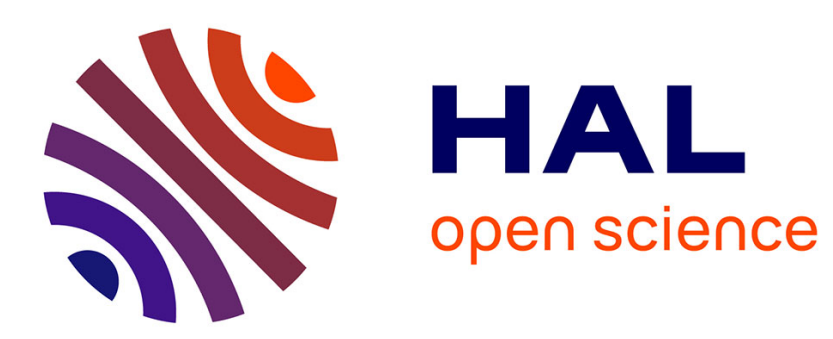

\title{
"La Zone Xtrême " et ses publics télévisés : situations de communication multiples et ajustements permanents
} Camille Jutant

\section{To cite this version:}

Camille Jutant. " La Zone Xtrême " et ses publics télévisés : situations de communication multiples et ajustements permanents. Communication \& langages, 2010, 2010 (166), pp.101. hal-02005942

\section{HAL Id: hal-02005942 \\ https://hal.science/hal-02005942}

Submitted on 8 Apr 2019

HAL is a multi-disciplinary open access archive for the deposit and dissemination of scientific research documents, whether they are published or not. The documents may come from teaching and research institutions in France or abroad, or from public or private research centers.
L'archive ouverte pluridisciplinaire HAL, est destinée au dépôt et à la diffusion de documents scientifiques de niveau recherche, publiés ou non, émanant des établissements d'enseignement et de recherche français ou étrangers, des laboratoires publics ou privés. 


\section{«La Zone Xtrême » et ses publics télévisés :}

\author{
situations de communication multiples \\ et ajustements permanents
}

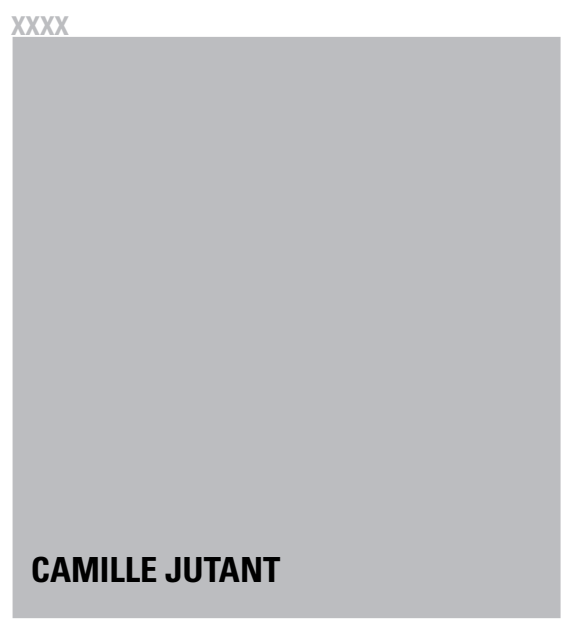

Cet article présente les résultats de l'enquête menée sur le plateau de la fausse émission de jeu "La Zone Xtrême ". II analyse les situations dans lesquelles a été impliqué le public en prenant en compte la spécificité du dispositif télévisuel en tant que dispositif de communication. La redéfinition des situations (jeu - expérimentation - documentaire) est interprétée par les individus à partir de leur culture médiatique et d'une profonde réflexion sur les enjeux des situations médiatiques aujourd'hui. Alors que la querelle a opposé un public dupe et un public non dupe, cet article montre que les publics opèrent un ajustement au dispositif télévisuel, en convoquant des représentations sur le jeu et la fiction télévisés ainsi que des figures du public des spectacles médiatiques. La prise de rôle et l'incertitude sur la nature des situations constituent ainsi l'expérience la plus structurante pour ces publics.

Mots-clés : publics, ajustement, dispositif télévisuel, figures de public, interprétation, corps, culture médiatique, fiction, jeu télévisé 
des situations médiatiques (au moment du spectacle en studio, au moment de sa médiatisation et de sa diffusion).

Dès le 8 mars 2010, il est question du public de l'émission « La zone Xtrême ", dans les articles de presse qui ont précédé et suivi la diffusion du documentaire Le Jeu de la mort consacré aux dérives de la télévision, mais aussi dans les contributions postées sur le forum de france2.fr Jusqu'où va la télé ?². Plusieurs personnes s'interrogent : «Et le public ?» «Personne ne parle du public !". Néanmoins la question que pose ce public semble déjà claire pour tout le monde : en mobilisant un public, l'expérience " La zone Xtrême » introduit une autre composante par rapport à l'expérience de Milgram, qu'elle prétend reproduire ${ }^{3}$. Dans l'ensemble des commentaires, le public incarne tour à tour la figure de la masse oppressante dénuée de conscience morale, illustrée par l'expression « les moutons de panurge ${ }^{4}$, et la figure d'un acteur responsable en ce sens qu'il est doué d'un désir et de la capacité à l'exprimer. "L'effet de ce public, et l'impact qu'il a sur un candidat avide de jouer le rôle du «bon candidat » en se conformant au désir du public, est au moins aussi important que la soumission à l'autorité incarnée par l'animatrice $»^{5}$. Le public de l'émission devient vite un fantôme qui circule dans les discours médiatiques et qui semble convoquer à nouveau les analyses de Gabriel Tarde et Gustave Le Bon qui opposaient, au début du $\mathrm{XX}^{\mathrm{e}}$ siècle, le public à la foule ${ }^{6}$.

Travailler sur les publics de l'émission de jeu « La Zone Xtrême », c'est avoir l'occasion d'interroger en profondeur le statut de ces publics télévisuels, tant le tournage de cette émission les engage déjà dans une situation complexe, du point de vue communicationnel. En effet, les publics de cette fausse émission se retrouvent confrontés à une redéfinition de la situation de communication dans laquelle ils sont impliqués. Entre le 14 et le 24 avril 2009, ils viennent participer au tournage d'un pilote de jeu télévisé, au studio 107 de la plaine Saint-Denis et, une fois que le premier candidat est passé, les membres de l'équipe de tournage leur expliquent qu'ils assistent en réalité à une expérience scientifique qui fera l'objet d'un documentaire sur le pouvoir de la télévision. On leur demande alors de bien vouloir continuer à jouer le rôle d'un public de jeu tout en sachant que les candidats, eux, ne sont pas au courant de l'expérience. En d'autres termes,

2. Le 25 avril 2009, Libération proposait déjà un dossier consacré au tournage de l'émission, dans lequel le rôle du public était mentionné et questionné.

3. Milgram, S., 1974, Soumission à l'autorité : un point de vue expérimental, Calmann Levy.

4. On peut lire aussi par exemple : « la foule, cette bête immonde, fonctionne toujours sur le même principe, une mise en commun de la bêtise humaine " (forum). De nombreuses expressions de ce type révèlent une représentation du public en tant que figure historique de l'aliénation des consciences individuelles.

5. Contribution de TocquevilParis, 17 mars 2010 à $17: 11: 26$.

6. Témoin des changements sociaux, politiques et économiques de la fin du XIX ${ }^{\mathrm{e}}$ siècle, Gustave Le Bon explique que le groupement d'individus en foule produit une nouvelle réalité dans laquelle l'individu régresse et est ramené à ses instincts les plus primaires. Pour Gabriel Tarde, le public est d'abord composé par une somme d'individus, il est « une collectivité purement spirituelle, comme une dissémination d'individus physiquement séparés et dont la cohésion est mentale » (p.8) et il implique un degré de maturité important. Le Bon, G., 2003 [1895], Psychologie des foules, PUF, "Quadrige ", Paris et Tarde G., 1901, L'opinion et la foule, Alcan, Paris. 
ils assistent à un brouillage entre la " promesse ontologique » d'un jeu télévisé et les «promesses pragmatiques » que tiennent simultanément les acteurs du jeu (l'animatrice, le chauffeur de salle, les cameramen) et ceux du documentaire (le faux producteur, les chercheurs) ${ }^{7}$.

La question sur laquelle je travaille est celle de l'ajustement des individus par rapport aux dispositifs médiatiques. La raison pour laquelle la situation du faux jeu «La Zone Xtrême » a requis l'analyse ici menée est l'occasion qu'elle offre de décrire et d'analyser la façon dont les membres du public ont engagé et négocié des espaces d'interprétation à l'intérieur d'une situation extrêmement déroutante. Cette analyse appuyée sur le concept d'ajustement permet de rendre compte de la grande richesse de la réflexivité des sujets sociaux, engagés dans un dispositif de communication. La demande de la production était de mener une analyse des publics mais nous avons décidé que celle-ci ne pouvait prendre sens qu'au sein d'une analyse sémiologique du dispositif du plateau de jeu et de la place qu'il engage pour les différents acteurs. Pour notre équipe de chercheurs ${ }^{8}$, il s'agissait de confronter une situation médiatique (celle du faux jeu) avec les activités d'interprétation produites par les acteurs engagés dans cette situation. Nous avons essayé de travailler à partir d'un principe méthodologique, qui était précisément de ne pas poser un cadre interprétatif unique, lié aux enjeux psychosociologiques de l'expérimentation (rapport de soumission à l'autorité), pour analyser les discours et les pratiques des membres du public. Je propose cette analyse à partir de trois corpus de documents : les cahiers de notes et les entretiens réalisés à l'occasion des journées d'observation participante auprès des publics pendant le tournage de la fausse émission de jeu ; des entretiens réalisés par l'équipe de réalisation dans les gradins, auprès des membres du public ${ }^{9}$ et enfin les discours des internautes sur le forum de france2.fr consacré au documentaire et mis en ligne en février 2010. J'ai aussi eu accès aux résultats de l'enquête par questionnaire qui a été réalisée auprès des publics pendant toute la durée du tournage et que je citerai dans la dernière partie de cet article.

7. François Jost propose le terme de "promesse " pour qualifier la nature la relation communicationnelle qu'engagent le document audiovisuel et sa diffusion avec ses récepteurs; Cette promesse est celle " d'une relation à un monde dont le mode ou le degré d'existence conditionne l'adhésion ou la participation du récepteur» (Jost, F., 2007 "Ruptures et retournements de la sémiologie des médias à l'ère de la communication ", Semen [Online], 23, mis en ligne le 22 août 2007. URL : http://semen.revues.org/5091, p.19). Elle est " un acte unilatéral qui n'oblige que le locuteur » et qui " entraîne chez le spectateur des attentes que la vision du programme met à l'épreuve ". Ces attentes sont composées de croyances, de savoirs associés aux genres audiovisuels existants et de savoirs " propres à la relation de l'image à son objet » (Jost, F., 1997, « La promesse du genre », Réseaux, 81, p. 7).

8. Yves Jeanneret a piloté la recherche sur les publics et sur les conditions de l'interprétation pour les acteurs engagés dans le faux jeu. Cet article s'appuie sur les observations menées ensemble pendant le tournage.

9. 440 entretiens filmés ont été réalisés auprès du public entre le 14 et le 24 avril 2009. Ces entretiens n’ont pas été menés par notre équipe néanmoins la grille de questions utilisée aborde le rapport entre interprétation et situation de communication; ces entretiens se révèlent d'une grande richesse pour la compréhension des interactions entre les membres du public et le dispositif. Un corpus de 65 entretiens a été sélectionné pour une analyse détaillée. 36 d'entre eux sont réalisés auprès du public en situation dite « naïve ». Je remercie Camille Robert qui m'a facilité l'accès aux données. 


\section{Construire un statut de public}

Le questionnement autour du public de "La Zone Xtrême » débute avec un constat : ce public est plongé dans une tension entre les deux ressorts principaux du dispositif (être à la fois une réplique de jeu télévisé et une expérience scientifique). En d'autres termes le public est pris entre la participation à un protocole scientifique d'expérimentation - qui engage la réapparition du faux candidat, la répétition et la régularité des comportements des acteurs sur le plateau afin que les résultats soient comparés - et la fonction d'attester une situation de jeu télévisé qui requiert un public pour que le dispositif soit une réplique exacte de jeu. Il s'agit ici de la norme infra-ordinaire du fonctionnement des jeux télévisés. La situation de jeu télévisé engage à son tour une double fonction pour le public : il doit être un acteur « exhortant » pour le candidat (c'est le cadre du jeu), mais aussi une masse compacte et télégénique, en arrière-plan de l'action (c'est le cadre télévisé).

Deux niveaux d'analyse s'offrent ici en ce qui concerne la place du public : d'une part, un niveau manifeste, qui est celui de l'enrôlement du public dans une série de 4 séquences de jeu apparemment identiques mais sous-tendues, en fait, par des hypothèses de réalité différentes (avec le premier candidat, le public pense assister à un pilote de jeu télévisé, puis à partir du second candidat, il assiste à une expérimentation scientifique). Mais d'autre part, il existe un niveau plus profond, qui est celui d'une surdétermination, dans toutes les séquences, d'une prise de rôle pour ce public (il joue le rôle du public pour le pilote, puis il joue le rôle du public pour le candidat).

Comment caractériser alors la pratique à l'œuvre de ces publics quand elle est elle-même qualifiée plusieurs fois et dans plusieurs espaces, en tant qu'agir stratégique et dramaturgique ${ }^{10}$ ? Bien que la querelle, analysée dans les articles d'Yves Jeanneret et de Valérie Patrin, exige de trancher entre un statut "naïf " ou un statut « lucide », cet article veut montrer qu'en réalité les perceptions de la réalité de la situation flottent, pour le public, avant comme après l'élucidation de l'expérimentation. Il ne s'agit pas ici dire si le public est dupe ou n'est pas dupe. Notre position est d'essayer de comprendre les pratiques du public en tant qu'elles relèvent d'une situation médiatique qui mobilise précisément la figure du public comme une figure signifiante.

Rappelons que plusieurs héritages théoriques sont ici mobilisés. Le fonctionnalisme américain et les études culturelles des années 1960, ont permis d'affranchir la relation entre téléspectateurs et objet télévisuel, d'un rapport mécanique d'adhésion et ont ouvert la voie vers la prise en compte des médiations symboliques et des phénomènes de mise à distance critique ${ }^{11}$. Ces travaux étudient la façon dont les individus téléspectateurs produisent du sens à partir des programmes en tant qu'ils sont des textes $^{12}$. La notion de public est explorée. John Hartley critique,

10. Habermas, J., 2001 [1987], Théorie de l'agir communicationnel, Tome I, Rationalité de l'agir et rationalisation de la société, Fayard, Paris.

11. Pour un aperçu des grands axes autour des travaux sur la réception, voir Esquenazi, J.P., 2003 Sociologie des publics, La Découverte, "Repères "; et Proulx, S. (dir), 1998, Accusé de réception, le téléspectateur construit par les sciences sociales, L’harmattan, coll. «Champs visuels », pp. 121-161.

12. Hall, 1997 [1980], " codage/décodage », Sociologie de la communication, CNET, Paris ; et Ang, I., 1991, Watching Dallas, Routledge, Londres. 
à ce titre, le fait que le public ou plutôt les communautés d'interprétation soient une pure invention du chercheur ${ }^{13}$. Il semble, montre-t-il, qu'il n'existe pas de groupe social constitué comme le public a priori de tel ou tel programme. Les publics existent réellement comme des représentations opposées à d'autres représentations. Il existe un "processus de construction socio-symbolique des « publics » qui est à l'œuvre autant dans les pratiques de programmation et de désignation des «publics " par les gestionnaires de la radiotélévision que dans les pratiques des chercheurs ${ }^{14}$. Joëlle Le Marec, dans ses recherches sur les publics des musées, montre que ces représentations circulent et finissent par intégrer le sens commun ${ }^{15}$. Daniel Dayan parle à ce titre de « réflexivité de la notion de public». Les travaux de Fiske démontrent bien que le fait d'être public est davantage une activité qu'il nomme "audienciation » (le fait de prêter son attention, de se constituer en spectateur) qu'un état ${ }^{16}$. Or les recherches qui cherchent à comprendre ce qui détermine l'audienciation expliquent qu'il y a une négociation chez le spectateur entre différentes figures de public (celles proposées par le programme de télé, mais aussi celles proposées par les discours circulants sur la télévision, et enfin celles que le spectateur anticipe ${ }^{17}$. "Au lieu d'être posée en acteur de la réception, la notion de «public » serait l'un des contenus lui-même soumis au procès de la réception $»^{18}$.

\section{Une problématique de l'ajustement}

Cette perspective permet de poser l'axe qui sera le nôtre dans cet article et qui considère l'importance du travail interprétatif des membres du public. Rappelons que Roger Odin désigne le public comme " un ensemble d'individus réunis par la mise en œuvre d'un système de modes de productions de sens $»^{19}$. La notion de public implique dès lors une réflexion qui porte à la fois sur les conditions de réception des objets et sur l'existence possible d'un horizon d'interprétation collectif. On privilégie à ce titre une analyse sémio-pragmatique des situations de réception ${ }^{20}$. Le public ne construit pas seul le sens qu'il souhaite donner à la situation de communication. Il est toujours engagé dans une situation qui lui résiste et qui convoque des figures interprétatives spécifiques. Ces figures sont saisies par l'individu qui engage un travail d'interprétation. Je propose de qualifier cette situation de réception un « ajustement au dispositif ». Il ne s'agit

13. Hartley, J., 1988, « The Real World of Audiences », Critical Studies in Mass Communication, 5.

14. Odin, R., 2000, « La question du public. Approche sémio-pragmatique », Réseaux, vol. 18, 99.

15. Le Marec, J. 2007, Publics et musées, la confiance éprouvée, L’harmattan.

16. Fiske, J.,1992, « Audiencing: A cultural Studies Approach of Watching Television », Poetics, 21.

17. Soulez G., 2004, « Nous sommes le public ». Apports de la rhétorique à l'analyse des publics, Réseaux, 126.

18. Dayan, 1998 [1993], "Le double corps du spectateur », in Proulx, S., Accusé de réception, L'harmattan, coll. «Champs visuels », p. 184

19. Odin, R., 2000, «La question du public. Approche sémio-pragmatique », Réseaux, vol. 18, 99, p. 60.

20. La sémio-pragmatique s'intéresse en priorité aux grandes modalités de la production de sens et d'affects ainsi qu'à leurs conditions de mise en œuvre. Le contexte est un opérateur fondamental dans la production du sens et dans la définition de la situation de communication. 
pas d'un ajustement à l'autorité mais d'un ajustement à l'espace médiatique et qui implique la construction, pour l'individu, d'un rapport plus général aux dispositifs de communication, aux images, aux institutions. Dans l'émission « La Zone Xtrême ", la réévaluation des paramètres du jeu va permettre de rendre compte de ce processus pour le public : un ajustement vis-à-vis de la situation médiatique et un retour réflexif sur le propre engagement de l'individu. Je tenterai de montrer que la perplexité que suscite la situation devient un opérateur de cet ajustement, c'est-à-dire qu'elle est au fondement de la relation qui se noue entre l'engagement dans la situation spécifique et la construction d'un rapport plus général aux médias.

Je proposerai trois points d'analyse : la circulation des figures $d u$ public associées au dispositif de jeu, dans le documentaire et le débat public; l'analyse de la situation de communication dans laquelle se trouve engagé le public de la fausse émission; et enfin l'analyse des éléments interprétatifs mobilisés dans le travail d'ajustement des membres du public.

\section{LE PUBLIC EST UNE FICTION}

Dans cette partie, je propose de regarder comment le public en tant que figure signifiante circule et se fixe depuis le tournage de l'émission, la diffusion du documentaire et la querelle. Le corpus de témoignages n'est pas exhaustif, il s'agit au contraire de mettre en exergue plusieurs exemples, à partir des images du documentaire et des discours dans les forums, qui composent ainsi un portrait bigarré de cette figure télévisuelle et posent avec acuité la question de son statut.

\section{Que fait le public ?}

Les participants du forum de France 2 notent que la situation du public n'est pas explicitée dans le documentaire. Les internautes formulent des hypothèses sur le statut du public et leur engagement dans l'expérience : sont-ils au courant ? De quoi ? À quel moment ? Sont-ils complices ? Cette incertitude sur le rôle du public laisse planer une incertitude sur le reste du film. «Je pense ici que le problème du public est biaisé, ou que ça montre que tout est truqué !» ${ }^{21}$

Le documentaire, en effet, n'explicite pas les conditions réelles de participation du public. Ce dernier semble plutôt un acteur ignorant les enjeux réels de la situation à laquelle il assiste. "Comme dans tout divertissement, ils ne savent rien ! $»^{22}$. Dès les premières minutes du documentaire, néanmoins l'entité « public » apparaît sous des traits qui ne sont pas anodins. Il s'agit de publics d'émissions étrangères dans lesquelles les candidats subissent diverses souffrances. Dans la suite du documentaire, le public est métaphorisé à plusieurs reprises, sous les traits de la masse (les stades remplis de spectateurs, les foules dans la rue). Dans les plans qui montrent le faux-réalisé (l'émission " La Zone Xtrême »), on voit le public tour à tour riant ${ }^{23}$, applaudissant, scandant " fortune, châtiment »,

21. Contribution de Lefigolu : 18 mars 2010 à 11:19:21.

22. Minute $14: 00$.

23. Minute $01: 49$. 
mais aussi perplexe, attentif, les yeux rivés sur le candidat ou les yeux cherchant le chauffeur de salle ${ }^{24}$. En réalité, le public est tout de suite un acteur essentiel de la situation de communication : on entend sa voix, on voit son regard qui témoigne pour tous les téléspectateurs d'une réalité bel et bien observée et vécue.

\section{Témoin ou injonction?}

Néanmoins, lorsque le documentaire annonce le parallèle avec l'expérience de Milgram et insiste sur le parallélisme des protocoles, il efface la spécificité du dispositif médiatique et du statut du public. Il faut attendre la minute 13 pour entendre : « Pour que l'expérience soit crédible, il faut qu'elle se déroule en public; c'est la seule différence d'avec l'expérience de Milgram qui s'est déroulée dans l'isolement d'un laboratoire de recherche. » À ce moment-là, le public surgit à l'écran ${ }^{25}$ déjà ensemble, déjà assis, comme un déjà-là de la situation télévisuelle ${ }^{26}$. Dès lors, le public apparaît presque toujours comme une masse devant laquelle se détache un face-à-face entre la présentatrice et le candidat. La situation de ce public dans le documentaire est intéressante : le public est toujours "regardé », mais jamais « regardant » à une exception près, lorsqu'il est mention de l'injonction : la focalisation se fait à partir de l'arrière des gradins, depuis le point de vue du public. Ainsi, le documentaire attribue trois fonctions au public : il est à la fois un décor, un acteur dont on attend de voir la réaction « Jusqu'où ira le public ? » et une injonction. En effet, Jean-Léon Beauvois explique que le public représente la $5^{\mathrm{e}}$ injonction et qu'elle était censée être «la plus pesante».

Le statut de cette injonction n'est pas perçu de la même façon par les différents acteurs enrôlés dans le débat. Les psychosociologues ont pu conclure que le public n'avait en réalité que peu d'influence sur la décision du candidat lorsqu'il décide d'arrêter le jeu. Or on peut considérer, qu'à la $5^{\mathrm{e}}$ injonction, la posture de refus est déjà construite et affirmée chez le candidat. En réalité, le public est une donnée, dès le départ, plus qu'une injonction. Il est présent dès que le candidat rentre sur le plateau. Lors de la rencontre avec le faux producteur qui explique les règles du jeu au candidat, l'un d'eux dit « je vais me faire huer $!{ }^{27}$, puis au moment de rentrer sur le plateau « je suis un peu stressé. Il y a trop de monde là » ${ }^{28}$. Le public accueille le candidat.

\section{Un agent coupable?}

Est-il responsable de ses actions ? Il est très intéressant d'observer que le statut de public est considéré, dans les commentaires, comme un rôle dont les membres auraient pu se dessaisir pour la rejeter. L'adhésion au statut de public ferait d'eux les responsables de l'horreur au même titre que le questionneur. « Tout le

24. Minute $03: 24$.

25. Minute $13: 36$ du documentaire.

26. Le documentaire insiste aussi sur le caractère authentique de ce public : " nous ne voulions pas de figurants. Nous voulions aussi savoir si un vrai public de jeu tolèrerait un vrai spectacle. » (minute $13: 48)$.

27. Minute $12: 00$ du documentaire.

28. Minute $42: 59$ du documentaire. 
monde est complice ». Le terme de " public complice » est repris par Christophe Nick lorsqu'il explique que les membres du public sont informés de la situation d'expérience et exhortés à continuer à jouer leur rôle. Néanmoins, c'est dans un autre sens que le terme est employé, celui de complice des scientifiques et non pas complice de l'horreur. De nombreuses personnes, dans la presse et dans le forum, ont parlé d'état agentique pour dénoncer l'attitude du public : il est par ailleurs désigné comme " obéissant ", " bourreaux ", " soumis ». Il est coupable de son comportement. À ce titre, un épisode sur le forum est tout à fait révélateur de l'instrumentalisation croisée de la figure de public omnipotente, la responsabilité éthique et les représentations qui sont mobilisées sur la discipline psychanalytique.

Une internaute $^{29}$ intervient le 17 mars 2010 à $22 \mathrm{~h} 48$. Elle relaie sur le forum le texte qu'elle a envoyé au journal Télérama. Elle critique la parution du numéro 3139 et récuse les représentations qui y circulent sur le tournage de l'émission et le rôle du public dont elle faisait partie. En réalité, elle propose une critique du sensationnalisme qu'usent le journal et le documentaire, et répond à l'argument de la passivité du public et la manipulation dont il aurait fait l'objet. En utilisant un "nous, public » tout au long du passage, elle s'identifie aux autres membres du public, et fait du collectif un être sémiotique incrédule et doutant de la situation de jeu. Dans le forum, on lui rétorque que justement, si elle savait que c'était truqué, elle aurait dû partir. Elle semble même, pour les internautes, d'autant plus coupable qu'elle affiche cette sagacité vis-à-vis du trucage. «Tu es donc encore plus coupable que ceux qui n'ont rien vu... Toi tu es donc $200 \%$ bourreau. ${ }^{30}$ "Vous vouliez comprendre ce qui se cachait mais vous aviez tout compris. C'est un double langage... $»^{31}$. On lui explique que toute tentative de justification est un syndrome de " déni », argument, s'il en est un, implacable, et donc relativement insupportable. Cet argument d'autorité est un très bel exemple de représentations sociales de concepts freudiens telles que Moscovici les a justement étudiées ${ }^{32}$. La représentation est si forte qu'elle autorise les sujets qui l'emploient à s'en servir pour expliquer le comportement de ceux qui seraient incapables de donner le sens de leur action. La conclusion que tirent les internautes critiques est sans doute logique dans la mesure où elle est le fruit d'une réflexion dans laquelle le public est uniquement traité en tant qu'être psychologique.

Mais dès lors qu'on étudie le public comme l'interprétant d'une situation, c'est-à-dire qui construit du sens à partir des éléments spécifiques enrôlés dans la situation, on ne peut plus le qualifier unilatéralement de soumis, car il n'y a pas qu'une seule hypothèse de réalité binaire (croire/ne pas croire à la torture, se soumettre/ne pas se soumettre), mais des hypothèses complexes de jeu, de film, d'expérimentation qui engagent différents régimes de croyance. À bien regarder le documentaire, on se rend compte qu'il va dans ce sens : il offre en réalité une

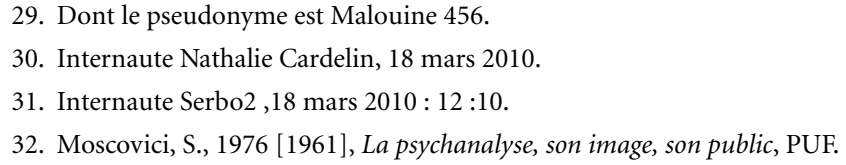


surcharge interprétative, en d'autres termes, une sur-sémiotisation oblique. Cela veut dire que si le protocole de lecture psychosociologique implique chaque geste code une attitude spécifique, on se rend compte, en réalité, que lorsqu'on filme la situation médiatique (candidat + public + plateau + animatrice) de nombreux petits événements apparaissent qui échappent au codage systématique. Tout au long du film, on observe par exemple des signes de surface qui ne sont pas traités, c'est-à-dire qu'ils ne servent pas à illustrer les étapes de la démonstration de la transposition de l'expérience de Milgram, mais qui renvoient néanmoins vers une autre histoire possible. Les scènes sont chargées de la richesse et de la complexité interprétative des membres du public. Les visages souriants sont parfois en décalage très fort avec la gravité des visages des candidats ${ }^{33}$ ce qui induit une dissonance interprétative. Un plan se place en porte-à-faux avec la représentation du public-masse. Un jeune homme est filmé à deux reprises, de près, se prenant la tête entre les mains ${ }^{34}$, engageant par là une réflexion sur le statut possible du public : est-il effaré ? Est-il complice ? On se doute alors que la situation dans laquelle se trouve le public est bien plus complexe qu'elle n'y parait.

\section{QUAND UN « PLATEAU télÉ » EN CACHE UN AUTRE : DISPOSItIFS ET CORPS PUBLIC}

\section{L'enchâssement des dispositifs}

Afin de comprendre dans quelle situation est véritablement impliqué le public de " La Zone Xtrême », il est indispensable de décrire le dispositif de l'émission, à partir de l'ambiguïté communicationnelle dont il fait l'objet, c'est-à-dire de réintroduire la spécificité de la situation médiatique. Les membres du public sont engagés tour à tour et de façon explicite dans plusieurs situations, dans lesquelles leur rôle, le cadre et la logique de communication évoluent. Certaines de ces modifications sont visibles et nommées (par exemple la distinction " naïf »/ " informé »), mais d'autres, plus essentielles et renvoyant plus largement à une culture médiatique, restent mobilisées implicitement. L'analyse sémio-pragmatique associée à une observation participante permet de les mettre en évidence. Il faut donc reprendre en détail les paramètres de la situation de communication pour mettre en évidence ce qu'elle engage pour les membres du public.

\section{Le cadre d'un spectacle ludique télévisé}

Le public est recruté pour participer à un jeu. L'équipe de réalisation a besoin de cent personnes par demi-journée d'enregistrement. Une demi-journée correspond au passage de quatre candidats. Au total, deux mille personnes sont invitées à participer au public de l'émission ${ }^{35}$. Deux sociétés spécialisées dans le recrutement des publics de télévision ont tout d'abord fait appel à leurs propres bases de

33. Par exemple minute $33: 58$ du documentaire.

34. Minute $32: 59$ du documentaire.

35. Les résultats de l'enquête par questionnaire révèlent que le public était composé de $65,3 \%$ de femmes et $28,2 \%$ d'hommes. La majorité a moins de 25 ans $(64,4 \%)$. On note que la deuxième tranche d'âge significative est celle des 50-64 ans (10,3\%). 
données de publics habitués des émissions de télévision. Elles ont aussi diffusé une annonce sur leur site internet, selon laquelle France Télévision préparait « un jeu de questions-réponses d'un genre très nouveau qui demande une forte implication de la part du public ${ }^{36}$. Enfin, elles ont procédé à des recrutements dans la rue en distribuant des invitations aux passants ${ }^{37}$.

Le cadre de ce spectacle engage une temporalité très spécifique. Le jeu télévisé est l'occasion d'une prise en direct pour les publics qui voient l'action se dérouler en temps réel sous leurs yeux. Par ailleurs, ce public atteste pour les téléspectateurs futurs que le jeu a eu lieu à un moment donné pour des gens qui l'ont bel et bien vu. La relation avec ce public de téléspectateurs est maintenue durant toute l'émission : l'animatrice s'adresse à eux régulièrement par le biais de la caméra. Et, par ailleurs, elle engage avec le public des gradins un partage de l'énonciation devant ces potentiels téléspectateurs (elle marque des pauses pour laisser le temps au public de scander ses interventions par des « ouahhh ", des «fortune » et " châtiment »). Dans cette situation, on peut dire que le public accepte de jouer un rôle, celui d'un ensemble d'individus qui s'exclame, s'esclaffe, s'étonne pour les candidats mais aussi pour un public à venir. Tout le monde sait que le public des émissions n'éprouve pas forcément les sentiments qu'il exprime mais qu'il s'agit de ressentis conventionnels. Une suspension volontaire de l'incrédulité est déjà au fondement du statut du public de jeu télévisé. Cette suspension se fait au nom des conventions et non de la fiction.

\section{Le tournage d'une émission pilote de jeu}

Cette émission est un pilote, le gain n'est pas réel, on fait "comme si » on allait gagner de l'argent. À ce titre, un pilote peut être considéré comme le mode fictif de l'émission. Tout public qui participe à un pilote accepte une fiction. Il suspend donc lui-même temporairement son incrédulité vis-à-vis du fait que ce n'est pas une vraie émission. Le dispositif du plateau prend en charge cette question en plongeant le public dans le jeu. Comme le note Laurence Leveneur, lorsque « le jeu est médiatisé, l'espace scénique dans lequel il se déroule, non seulement conditionne les performances des différents acteurs mais encore renvoie à des configurations symboliques qui régissent leurs interactions ${ }^{38}$. Le dispositif scénographique engage les membres du public à reconnaître une situation de jeu. À ce titre, le plateau de jeu fait l'objet de ce qu'on pourrait appeler un sentiment de familière étrangeté : le plateau télévisuel incarne un univers totalement étranger à celui dans lequel évolue le candidat dans sa vie quotidienne, mais à la fois, il en est familier, en a déjà vu à la télévision.

Le public est invité à s'installer sur trois gradins qui constituent l'arrière-scène du dispositif. On observe une structure concentrique marquée par l'enchâssement de 4 espaces : celui du public qui forme un U, celui de la console de jeu munie de manettes destinées à administrer des décharges électriques, à l’intérieur de laquelle

36. Annonce sur le site de Castings Events.

37. Nick, C., Eltchaninoff, M., 2010, L'expérience extrême, Don Quichotte, Paris, p. 61.

38. Leveneur, L., 2009, Les travestissements du jeu télévisé, Histoire et analyse d'un genre protéiforme, 1950-2004, Presses Sorbonne nouvelle, p. 131. 
est installé le candidat et à côté de laquelle se situe la présentatrice, qui introduit un face-à-face entre le candidat et la présentatrice ; celui de l'écran qui forme l'avantscène du dispositif et retransmet en direct le visage du candidat, inséré dans un cadre à côté duquel sont inscrites les questions à poser ; et enfin le sas avec une chaise électrique où est placé le faux candidat et que l'on ouvre et referme à chaque début de session de jeu. En ce qui concerne l'installation des membres du public, les équipes de production procèdent à un " assortiment » et les répartissent en fonction notamment de l'âge et du sexe. Une jeune fille, à côté de moi, me dit qu'on lui avait demandé de ne pas porter de rayures, "ça passe mal à la télé ! ».

\section{La participation à un jeu de questions}

Le principe du jeu est expliqué au début de la session : les candidats ont tiré au sort pour savoir qui allait poser les questions et qui allait répondre. Jean-Paul (le comédien) réitère à chaque fois sa surprise de se voir enfermé et de devoir répondre aux questions. Il devra retenir une liste de 27 associations de mots en une minute. Lorsque la minute s'est écoulée, le questionneur commence : il devra proposer, pour les 27 occurrences, une série de 4 paires de mots possibles ; une seule est correcte. À l'issue des 27 questions, les deux candidats se partageront 1000000 d'euros.

\section{Le spectacle d'une performance tournant au drame}

La situation du jeu de question évolue vers un exercice de torture. À chaque réponse incorrecte, le questionneur devra actionner une manette et administrer des volts à Jean-Paul, en commençant par 20 volts et en terminant par 460 volts. La console de jeu forme un arc de cercle. Elle tourne petit à petit devant le questionneur qui est installé de telle sorte qu'il est dos au public. La console, de quatre mètres de long, se compose de huit zones qui sémiotisent avec une gradation de couleurs vers le rouge et les chiffres des volts, l'intensité de la charge. Le public est dit " naïf» lors de la première occurrence de jeu. Il doit engager le joueur à continuer en criant « fortune » et « châtiment ».

La participation à une expérience scientifique sur les dérives de la télévision À l'issue du premier candidat, Jean-Paul va devoir réapparaître afin de jouer, à nouveau, le rôle du candidat que l'on enferme. Le public est donc mis au courant. Un des membres de l'équipe du tournage (le faux producteur, le chauffeur de salle ou un membre de l'équipe des chercheurs) lui révèle qu'il n'assiste pas à un jeu et lui explique le principe de l'expérimentation. Dès lors le public est considéré par les équipes de tournage comme "informé » ou « complice ». Voici un exemple d'une séquence d'élucidation menée par l'un des chercheurs en psychologie sociale :

Écoutez. Un petit peu d'attention. Alors pour vous expliquer un petit peu ce qu'il se passe... Tout d'abord il ne s'agit pas d'un véritable jeu télévisé. Il s'agit d'une expérimentation, une réplication, une reproduction d'expérience scientifique pour la télévision dans le cadre d'un jeu télévisé. Je fais partie de l'équipe scientifique des chercheurs en psychologie et ici on s'intéresse au pouvoir de la télévision et à l'autorité que peuvent représenter la télévision et la présentatrice dans le cadre d'un jeu télévisé. Donc ça ne passera jamais en tant que tel, en tant que jeu à la télévision 
mais c'est bien pour la préparation d'un documentaire sur la télé. Voilà. Donc ça, c'est la première des choses. Jean-Paul, bien sûr, n'a jamais reçu d'électricité, c'est un comédien. Tout cela est un enregistrement qui est préparé (...) et les candidats qui eux, venaient ne savaient pas tout cela, de la même manière que vous, vous ne saviez pas. ${ }^{39}$

Désormais ce que les membres du public observent, participe d'une double réalité. Les mouvements des acteurs sur le plateau « figurent et racontent » à la fois un récit de jeu (le vrai candidat) et un récit d'expérimentation (l'animatrice, le comédien). À ce titre, la sémiotique théâtrale ${ }^{40}$ rappelle que « le statut propre des signes de la représentation est double. Le signe [...] est à la fois signe d'autre chose, renvoyant à un quelque chose dans le monde qu'il signifie, et signe-pour-soi, élément d'une performance qui est une pratique spectaculaire », ce qui est rendu possible par le fait que le signe théâtral est homo-matériel à ce qu'il représente ${ }^{41}$. La scène de "La Zone Xtrême » devient pour les publics « un réel qui fait signe » et sa spécificité est que d'une part, les signifiés sont multiples (jeux, expérience) et que d'autre part, la superposition possible de ces différents signifiés tient à un équilibre très fragile entre le spectacle et l'expérimentation.

\section{Le tournage d'une expérience scientifique qui sera diffusée un jour}

Un autre élément de l'élucidation consiste à expliquer au public que cette expérimentation va faire l'objet d'un documentaire qui sera diffusé sur France 2. Il s'agit de réfléchir aux dérives de la télévision et de sensibiliser les téléspectateurs afin qu'ils rejettent les émissions qui orchestrent la violence à l'écran.

\section{L'enrôlement dans des entretiens avec des chercheurs et des cameramen}

Une nouvelle situation de communication s'engage dès lors que les membres du public sont sollicités individuellement pour répondre à quelques questions après les sessions de jeu. L'analyse de ces entretiens filmés révèle que le public y reconnaît une nouvelle situation de communication. "Attendez il faut que je me fasse une beauté » (extrait entretien 41). Le fait qu'un caméraman vienne les interroger dans les gradins a plusieurs conséquences : ils prennent la parole devant les autres membres du public ; ils hésitent entre une adresse à la lentille (le fameux regard caméra) et une adresse au caméraman qui se situe bien souvent au-dessus de la caméra ; ils utilisent le dispositif comme une tribune : "dédicace pour Sartrouville !». On observe en outre un phénomène analysé par Joëlle Le Marec pour les publics de musées ${ }^{42}$ : les individus identifient les cameramen comme des représentants de l'équipe de production du jeu - et à ce titre, cherchent bien souvent à lui faire plaisir ou à se faire rassurer sur la validité de leur réponse, « je

39. Retranscription d'une intervention de l'un des chercheurs chargés de l'élucidation auprès du public. 40. Anne Ubersfeld rappelle que " pour qu'il y ait espace théâtral, [il faut et il suffit] qu'il y ait des hommes unis par la fonction du regard : des regardants et des regardés [...] l'espace est défini par cette relation même » (Ubersfeld, A., 1996 [1981], Lire le théâtre Tome II. L'école du spectateur, Belin, Paris, p. 51).

41. Idem, p. 36

42. Le Marec, J. 2007, Publics et musées, la confiance éprouvée, L’harmattan, p. 62. 
ne sais pas si j'ai bien répondu » - et se construisent en contrepoint un statut de représentant du public soit de l'émission de jeu, soit du public potentiel de la télévision française.

\section{Le rapport du corps au dispositif}

Le plateau de jeu télévisé engage les membres du public physiquement : ils sont impliqués dans une situation de communication qui est aussi complexe du point de vue de l'engagement corporel qu'elle attend d'eux. Ce public est dans une situation où il doit se conformer de façon absolument collective à un certain nombre de gestes, qui lui sont « ordonnés » par le chauffeur de salle. Ces gestes observent deux caractéristiques liées au double statut de la situation : ils doivent ressembler à des gestes de jeu (reproduire les conditions d'un vrai jeu) et ils doivent être répétés à l'identique devant tous les candidats (régularité des protocoles de recherche).

Je voudrais rappeler que l'interprétation comme phénomène cognitif n'est dissociable ni d'un contexte d'interprétation ni d'une posture physique d'appropriation de la situation. "Le corps percevant, agissant et parlant est le médium de la compréhension, et c'est à travers les épreuves qu'il traverse qu'un sens émerge petit à petit ${ }^{43}$. Et inversement, comme l'a montré Howard Becker avec les recherches qu'il a menées sur les consommateurs de LSD, l'expérience est vécue en fonction des conditions de son interprétation ${ }^{44}$. Il s'agit donc ici de comprendre le corps en tant qu'il est une matière signifiante pour les autres avec qui il rentre en interaction. Et par ailleurs, il s'agit de comprendre le corps en tant qu'il est médiateur du rapport au dispositif pour l'individu lui-même. En d'autres termes, l'ajustement au dispositif passe d'abord par une appropriation et une incorporation du dispositif. Or celui-ci pose des conditions à l'incorporation. Yves Jeanneret propose le terme d' «implication » pour désigner « cette dimension du pouvoir-faire autorisé par la saisie pratique des productions médiatiques. [...] Distinct de l'idée de contrat, ce terme désigne le fait que le complexe média-texte engage pratiquement l'échange dans un certain sens et requiert le corps des sujets ${ }^{45}$. Nous allons voir maintenant comment les corps des membres du public sont impliqués dans le dispositif du jeu "La Zone Xtrême » qui les engage à un certain nombre de gestes signifiants, mais aussi à anticiper que ces gestes feront l'objet d'une diffusion à la télévision.

\section{La mise en scène des corps}

Le dispositif de la "La Zone Xtrême » introduit un rapport au corps qui est régi par un certain type de relation "voir/ne pas voir », à savoir l'opposition « caché/exposé ». Tout d'abord, le jeu repose sur l'exercice d'une torture infligée au faux candidat. Le rapport à son corps est ici un des ressorts dramatiques, les plus

43. Céfaï, D., (textes réunis, présentés et commentés), 2003, L’enquête de terrain, La découverte, «MAUSS », Paris, p. 544.

44. Becker, H., 1967, "History, Culture and Subjective Experience: An Exploration of the Social Bases of Drug-Induced Experiences", Journal of Health and Social Behavior, vol. 8, 3.

45. Jeanneret, Y, 2008 Penser la trivialité, volume 1, La vie triviale des être culturels, Hermès Lavoisier, Paris, p. 167 
importants du scénario. Ce corps est caché, on ne le voit pas, il est dans la cabine. Néanmoins, on entend ses cris, comme une métonymie de la souffrance vécue. On notera que ce corps en souffrance est très souvent montré dans les articles de presse et le documentaire (images de Jean-Paul attaché et qui hurle) alors que pas une seule fois il n'a été montré aux candidats et au public de l'émission au cours du tournage. À l'inverse, le corps du candidat est vu en «pleine vue » : il est au milieu du dispositif, il est vu du public dans les gradins, il est vu sur l'écran en face de la console de jeu, il sera vu à la télévision.

Un autre corps est exposé : le corps du public est vu par lui-même de deux manières : d'une part des moniteurs, au bas des gradins, filment les publics en permanence ; d'autre part les gradins sont installés face à face ; les membres du public sont assis face aux autres membres du public de l'autre côté du plateau. Le public reste tout le long de l'émission à cette place. D'une certaine manière il est en représentation de soi, en permanence, il n'a pas facilement accès à une zone de répit, une "région postérieure » pour reprendre les termes de Goffman, où il pourrait laisser tomber le masque ${ }^{46}$.

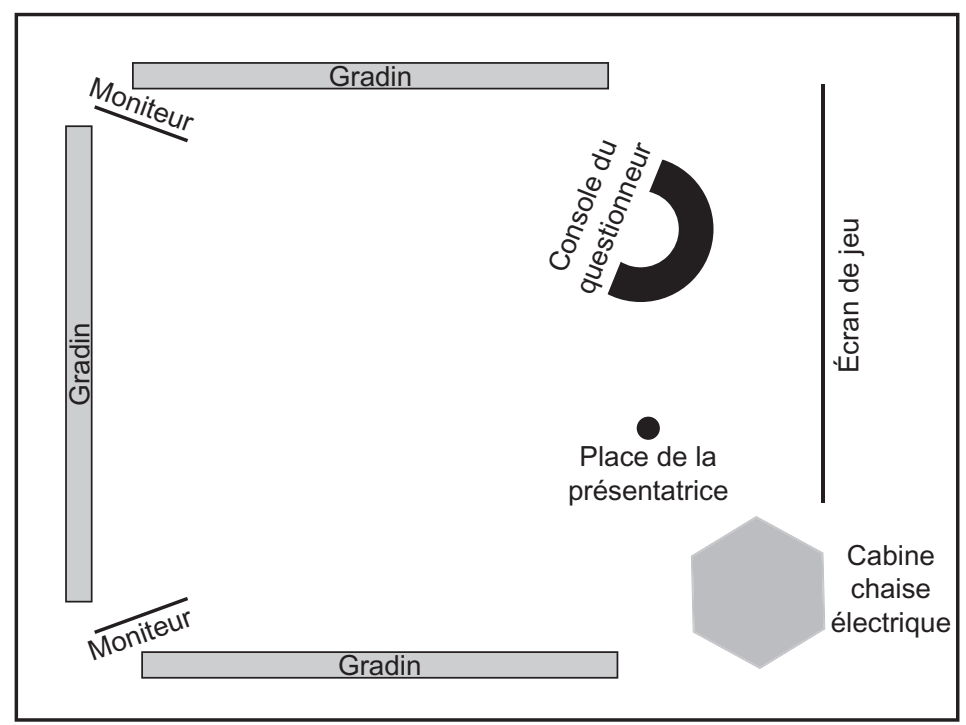

Figure 1 : schéma du dispositif du plateau de " La Zone Xtrême "

\section{Le corps docile}

Le chauffeur de salle indique au public ce qu'il doit faire pendant les sessions de jeu. Il l'exhorte à pousser de longs " ohhhh», « ahhh », à encourager le candidat (en l'occurrence, à crier " fortune ", "châtiment »). Le chauffeur de salle fait du public une entité signifiante (le bon comportement) pour les candidats et pour le jeu tout court, c'est-à-dire les futurs téléspectateurs.

46. Goffman, La mise en scène de la vie quotidienne, tome I, La présentation de soi, Éditions de Minuit, Paris, p. 105 
En faisant cela, le chauffeur de salle pousse le public à produire une image de soi conforme à un ethos collectif télévisuel. On est tenté de rappeler le texte de Foucault qui décrit la formation du rapport entre corps, obéissance et utilité. "Le corps ne devient force utile que s'il est à la fois corps productif et corps assujetti ${ }^{47}$. Dans le chapitre sur le corps docile du soldat, il décrit une rhétorique corporelle, porteuse de signes. "Le soldat est devenu quelque chose qui se fabrique ${ }^{48}$. Parce qu'il s'agit d'une expérience scientifique, la répétition et la régularité des comportements sont très importantes. Les membres du public sont soumis à une reproduction de leurs gestes de façon très stricte par le chauffeur de salle. C'est très intéressant de voir que lors de la première séquence de jeu, le chauffeur de salle laisse davantage les gens se parler. De fait, il y avait beaucoup de bruits quand j'étais dans le public. Or dès la deuxième occurrence (le public sait que l'émission est fausse), le chauffeur de salle demande expressément aux gens de se taire, de ne pas rire, pour ne pas révéler au candidat que l'émission est fausse (argument avancé par le chauffeur de salle lui-même).

\section{Sur-jouer son rôle}

Le chauffeur de salle s'appuie sur la connaissance qu'ont les individus des conventions liées au spectacle télévisuel, ce qui est rendu particulièrement visible après la révélation de la feintise. On se rend compte en effet que les membres du public se mettent à sur-jouer leur propre rôle. A posteriori, quand ils en parlent dans les entretiens, ils montrent qu'il existe une culture gestuelle dans laquelle on peut puiser pour savoir comment se comporter dans un studio. "Les analyses du geste font trop souvent l'impasse sur une telle influence constante des esthétiques médiatiques ${ }^{49}$. Ainsi nous avons observé une évolution notable dans le comportement des publics entre la première occurrence de jeu et les suivantes (c'est-à-dire entre la séquence 1 et les séquences 2,3 et 4 de jeu). L'excitation, la participation au jeu et les interactions entre personnes laissent peu à peu la place à une grande concentration et à une sur-ritualisation des comportements. "Lorsque j'arrive le public est déjà sur les gradins et il attend (il vient d'être chauffé) : ils sont déjà en train de bavarder et de s'agiter. Ils poursuivent, sans grand changement, cette attitude pendant la première séquence de jeu, pendant le remplissage des questionnaires, pendant le début de la seconde séquence de jeu. Mais cette agitation, déjà diminuée en début de séquence de jeu 2, disparaît totalement et ils assistent silencieux et stables à toute la séquence 2, à partir de

47. Foucault, M., 1975, Surveiller et punir, Naissance de la prison, Gallimard, «tel », Paris, p. 34. La référence au texte Surveiller et Punir, ne doit pas laisser penser que les publics sont ici unilatéralement assujettis. Au contraire, c'est la production d'un rapport signifiant (auquel participent tous les acteurs) entre le corps, la représentation qu'ont les individus de son utilité sociale (faire le public) et le jeu du chauffeur de salle qui est interrogée à partir du texte de Foucault.

48. (idem :159).

49. Kessler, I., 1996, "Corps et communication, le péril de Narcisse », Communication \& langages, 107, p. 96 et aussi, Courbet, D. et Fourquet, M.P., 2003, « Réception des images d'une catastrophe en direct à la télévision. Étude qualitative des réactions provoquées par les attentats du 11 septembre 2001 aux États-Unis au travers du rappel de téléspectateurs français ", European Review of Applied Psychology, 53 et Nick, C., Eltchaninoff, M., 2010, L'expérience extrême, Don Quichotte, Paris, p. 290. 
la troisième question posée » (cahier de notes en observation participante). Les individus se concentrent et exacerbent leurs gestes lorsqu'ils jouent au «public exhortant ». Le public s'ajuste au dispositif en se sémiotisant lui-même. Il devient signe de lui-même et produit ainsi une figure de public. Or, on se souvient des textes sur le paradoxe du comédien. «Les comédiens font impression sur le public, non lorsqu'ils sont furieux, mais lorsqu'ils jouent bien la fureur ${ }^{50}$. Interpréter un rôle revient à prodiguer aux autres les signes qui établissent l'intelligibilité de son rôle et ainsi à prendre une véritable distance vis-à-vis de son propre comportement. Pour Diderot, la nécessité de ne pas éprouver les sentiments lorsqu'on joue, est justifiée, d'une part par la mise à distance de la sensibilité, qui compromet le style, et d'autre part par la nécessité de convoquer des représentations communes de l'émotion qui est jouée. Lorsqu'on demande au public de « La Zone Xtrême » de continuer à faire comme avant, il s'agit pour lui de s'imaginer clairement un public pour lequel il joue (le candidat) et, d'autre part, de prendre de la distance vis-à-vis de son propre ressenti. Si l'on en croit Diderot, l'appel au paradoxe du comédien interdit de se demander à soi-même ce qu'on éprouve vis-à-vis du drame.

La question du rôle est donc présente tout au long de l'émission. Les rôles sont nombreux et ils changent, ils portent à la fois sur la vérité de la situation médiatique, la vérité des décharges et la vérité de la situation d'énonciation. L'opposition entre " authentique » et «feint », qui est convoquée dans la querelle ${ }^{51}$, masque que tous les acteurs qui participent à " La Zone Xtrême » sont, de toute façon, toujours engagés dans la production de la situation médiatique, et à ce titre que tout le monde participe à la fiction. Dès le début du tournage, un élément est central dans les conditions d'interprétation : avoir conscience d'un rôle à jouer. En d'autres termes, pour les membres du public, les modalités d'énonciation changent petit à petit et engagent différemment les acteurs du jeu, mais mobilisent toujours la fiction. Dans ce cadre, l'opposition entre public "naïf » et public "informé » est simplificatrice. Dans un premier temps, en participant à un jeu télévisé, le public se prépare à jouer un rôle à partir de conventions (que 100 personnes réunies puissent crier spontanément au même rythme, d'une seule voix) ; quand le public pense qu'il s'agit d'un pilote de jeu, il produit une fiction de lui-même ou plutôt une stylisation (que 100 personnes puissent faire croire que l'émission est une émission), puis il devient à la fois complice d'une feintise (après révélation) et énonciateur de cette feintise pour le candidat, en sur-jouant son rôle, c'est-à-dire qu'il est partie prenante d'une mise en scène de ses propres émotions vis-à-vis du candidat ${ }^{52}$. Cette façon de sur-jouer son rôle peut aussi être analysée ainsi : les publics manifestent de façon explicite qu'ils acceptent de s'enrôler dans la

50. Diderot, 1967 [posth, 1830], Le paradoxe sur le comédien, Garnier-Flammarion, p. 190.

51. Voir l'article d'Yves Jeanneret, dans ce dossier.

52. Kate Hamburger, dans son études des genres littéraires, et à sa suite François Jost pour l'analyse des genres des documents télévisuels, utilisent les termes de fiction et de feintise en distinguant la source de l'énonciation dans le récit, "Je-Origine réel » et "Je-Origine fictif ». Ainsi, la fiction produit une apparence de réalité, elle est " comme » la réalité mais elle est d'abord un monde autonome organisé par un « Je-Origine fictif », quand la feintise est " comme si » elle était réalité, elle renvoie à un probable " je-Origine réel ». Hamburger, K., 1986, Logiques des genres littéraires, Le Seuil, coll. " Poétique » et Jost, F., 1997, «La promesse du genre », Réseaux, 81. 
nouvelle situation de communication (participer à une expérience scientifique de dénonciation du pouvoir de la télévision).

Je propose un tableau récapitulatif. Il met en évidence la multiplicité des interventions qui participent à la redéfinition des situations ainsi qu'à leur interprétation. Il met également en évidence les décalages entre les différents acteurs dans la perception des mêmes situations.

\section{L'ENGAGEMENT COMMUNICATIONNEL DU PUBLIC}

Le rapport aux différents éléments clés de la promesse initiale se retrouve donc plongé dans une mise en abyme des situations de communication qui rend très complexes les cadres d'interprétation du public. Ainsi, après la révélation de l'expérience scientifique, les membres du public changent de posture. C'est le moment où ils vont commencer à sur-jouer leur rôle et où, en même temps, ils affirment une posture de doute. Le fait de désamorcer la feintise en l'exhibant n'entraîne pas une baisse de l'attention. Au contraire, le public est extrêmement concentré, dans l'attente de ce que sera le comportement du candidat. Il est plongé dans une profonde réflexion sur son propre statut. Les résultats de l'observation participante ainsi que de l'analyse des entretiens filmés vont dans le même sens : les membres du public sont désorientés ; ils interrogent profondément la situation dans laquelle ils sont engagés. Au fur et à mesure des redéfinitions de la situation, ils s'ajustent aux différents cadres en opérant un travail réflexif sur leur position. Je vais revenir sur les deux principaux éléments qui sont mobilisés dans le travail de réinterprétation des situations : l'incrédulité et le jeu ${ }^{53}$.

\section{L'insécurité sémiotique : la désorientation des publics}

«La situation créée par la confection du jeu, n'est pas un drame au sens pratique du mot, mais un drame herméneutique " (cahier d'observation). William Labov, parle d'insécurité linguistique pour désigner le comportement de l'individu qui se retrouve dans une situation dont il ne partage pas les compétences linguistiques ${ }^{54}$. En détournant sa formule nous parlons ici d'insécurité sémiotique pour qualifier le fait que les a priori diégétiques de l'émission sont bouleversés au cours du tournage et engendrent une crise de l'interprétation. De manière générale, les membres du public doutent, ils ne comprennent pas très bien la situation : il s'agit d'un faux jeu ? qu'est-ce qui est faux ? le documentaire ? pourquoi ? comment ?

Les situations dans lesquelles s'engage le public exigent d'eux à la fois la "suspension de l'incrédulité " ${ }^{55}$ (on leur demande de faire comme s'ils étaient dans un vrai jeu et de jouer des rôles) et la croyance (croire à la réalité des décharges puis croire à la réalité poignante que révèle l'expérimentation). Or si l'on accepte de jouer le rôle de celui, par exemple, qui scande « fortune - fortune » même si tout

53. J'exploite ici les observations et les entretiens furtifs que nous avons menés, pendant le tournage, mais les verbatims qui sont retranscrits sont extraits des interviews réalisées par l'équipe de réalisation. 54. Labov, William, 1972, Sociolinguistic patterns, University of Pennsylvania Press, p. 132

55. «La suspension volontaire de l'incrédulité » caractérise selon Samuel Coleridge l'attitude devant la fiction (on sait que l'histoire n'est pas vraie, mais on décide de faire comme si). Coleridge, S.T., 1985 [1817], Biographia Literaria, Princeton University Press. 


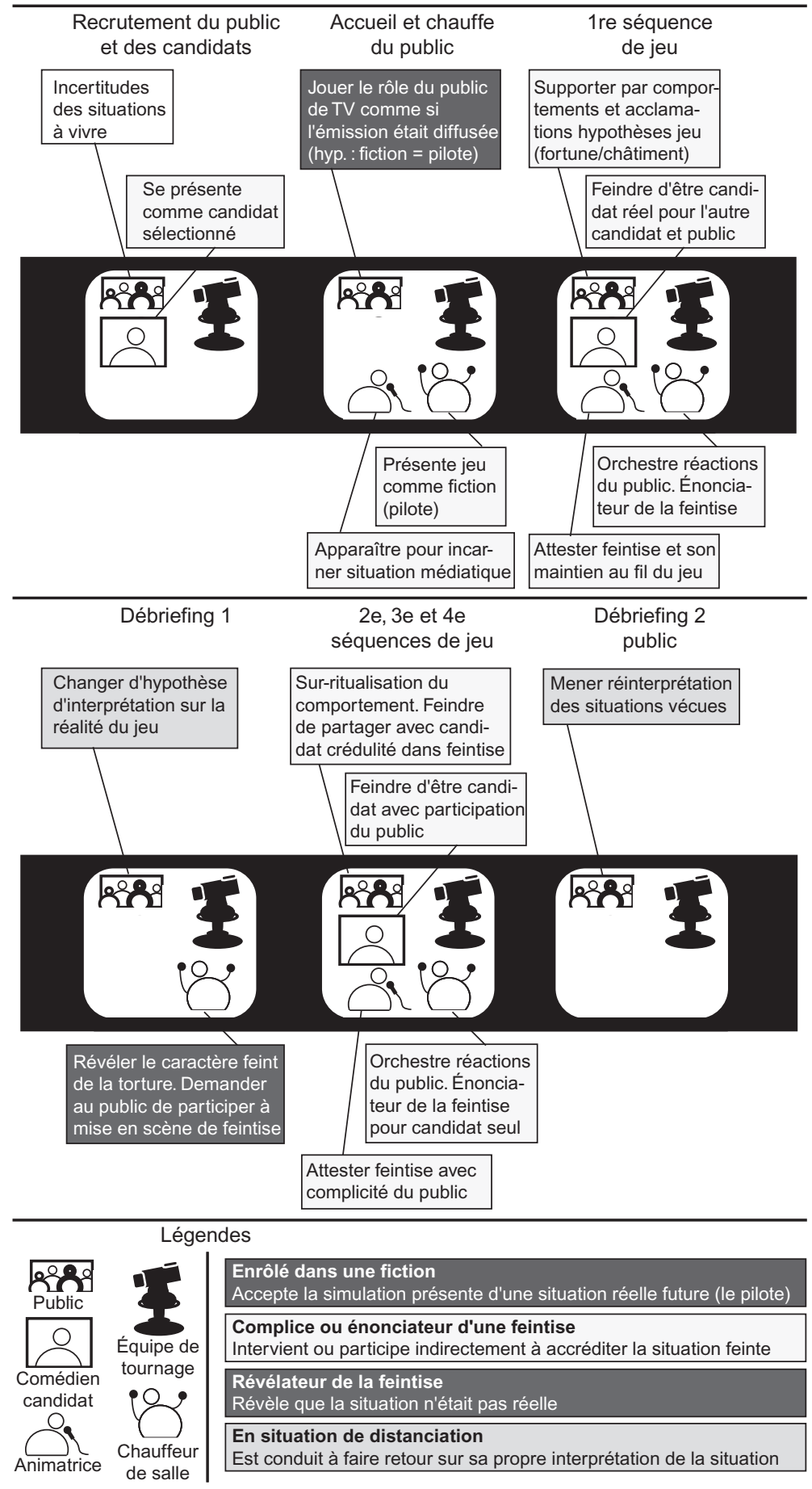

Figure 2 : les interventions des acteurs de "La Zone Xtrême " 
le monde sait qu'il n'y a pas de gain d'argent en jeu, pourquoi les autres données de l'émission seraient-elles vraies ? Les membres du public sont nombreux à identifier que la superposition des situations engage nécessairement un doute général sur la nature de la situation.

Nous avons observé de nombreuses contradictions, qui révèlent à la fois un engagement dans la fiction et un doute sur la possibilité d'un tel spectacle. Les hypothèses de plausibilité sont peu fréquentes mais elles existent ; ce genre de phrases par exemple : "Je suis contre la torture, même dans un jeu » (extrait entretien 1) révèle que les individus se positionnent contre un certain nombre de procédés qui pourraient faire irruption à la télévision.

Bah, le fait qu'ils reçoivent des chocs, on sait pas quand même ! bah, on y croit sans $y$ croire en fait ! (extrait entretien 41)

Au cours de la première séquence de jeu, le public hésite, doute et surtout s'interroge : «On s'est même plus qu'interrogés enfin au début, on s'interroge tout le temps, justement, bon, est-ce que c'est vrai ? est-ce que c'est pas vrai ? et après, à peu près au milieu des questions, on se dit : c'est faux quoi, et complètement impossible !» (extrait entretien 2). Un long entretien, qui a lieu dans les gradins, juste avant que la séance d'élucidation ne commence, révèle que les individus peuvent jongler eux-mêmes entre plusieurs hypothèses de réalité. Par exemple, le même interviewé explique qu'il pourrait jouer à " La Zone Xtrême » avec son ami bien que ce soit « dur de faire souffrir les copains » (hypothèse de souffrance) et que cette " émission qui fonctionne sur un certain voyeurisme et qui ne montre pas le type à l'intérieur fait que déjà on a du mal à y croire » (hypothèse de feintise). Le doute est une posture qui s'affirme tout au long du jeu et qui est nourrie par une incrédulité et une conscience de participer à un jeu.

\section{Incrédulité du public et culture médiatique}

Cette incertitude sur la nature de la situation est à la source d'une nécessité de recourir à une culture médiatique plus large. Les membres du public continuent ainsi à produire un ajustement. En effet ils sont préoccupés par le fait que le cadre de la situation est incertain, mais ils sont exhortés au cours des entretiens filmés à donner des réponses aux questions : Comment trouvez-vous l'émission ? Quels sont les points forts de l'émission? Quels sont les points faibles ? etc. On se rend compte que les membres convoquent alors un cadre d'interprétation plus large : celui des émissions de télévision, en général, du rapport à l'image, des rapports humains. On va voir par exemple qu'ils convoquent le plus souvent une représentation de la télévision française comme instance responsable.

\section{Un rapport de défiance et de confiance}

C'est intéressant de voir qu'il se joue à la fois une sorte de défiance (on reconnaît les mécanismes observés par Hoggart et Weissberg ${ }^{56}$ ) et de grande confiance envers

56. Hoggart, Richard, 1970, La culture du pauvre : étude sur le style de vie des classes populaires en Angleterre, Minuit, [1957] et Weissberg, 1999, "Dispositifs de croyance ", in Le dispositif, entre usage et concept, Hermès, 25. 
le dispositif télévisuel. Comme le dit Jean-Louis Weissberg, " un certain régime de vérité est révolu : celui par lequel l'image enregistrée lors d'une capture directe renvoyait au caractère apparemment immuable du passé »; il parle de crise de légitimité de l'audiovisuel dont la spécificité est le doute généralisé que suscite désormais toute image enregistrée ; "doute révélateur d'une séparation croissante entre le « voir » et le « croire » ${ }^{57}$ par rapport à une télévision qui « manipulerait » son audience (l'un des membres du public me dit que de toute façon « à la télévision tout est faux »). Par exemple le public estime que les candidats (aussi bien le vrai comédien que le vrai candidat) jouent des rôles et qu'ils les jouent mal. "Quelqu'un qui souffre, il ne crie pas comme ça, il ne continue pas à répondre aux questions » (extrait entretien 14). Ils sont considérés comme de piètres comédiens. Certains membres du public reconnaissent l'acteur qui joue le rôle de Jean-Paul. "Pour des acteurs, moi je les ai trouvés mauvais [...] Ils ne se sont même pas présentés à la base. Le type dans la bulle. . . il joue dans "faites comme chez vous !"» (extrait entretien 13)

Mais en même temps, le public fait état d'une confiance qui repose sur un contrat général de communication fondé sur la responsabilité éthique d'un service public. Ainsi, la télévision française ne tuerait jamais quelqu'un dans un jeu. «France Télévision n'envisagera jamais ce genre de programme ! (extrait entretien 17). Les cris, par exemple, ne sont pas crédibles « on ne crie pas comme ça à la télévision » (extrait entretien 13). À ce titre, "le son enregistré des réactions du faux candidat est diffusé à niveau sonore assez faible pour paraître crédible au vrai candidat, mais il est peu audible dans le public et pratiquement inaudible dans le quartier de gradins opposé à la " scène ». Il est intéressant de remarquer que c'est à ce type de tout petit détail qu'on voit que la superposition de la situation d'expérimentation et de la situation de médiatisation ne peut être parfaite. En effet, si l'on diffuse le son à un volume audible du public, il n'est plus crédible pour le candidat. Les deux ne sont donc pas tout à fait dans la même fiction. " (cahier d'observation)

Les décharges électriques sont évidemment un élément très important dans l'analyse que font les membres du public. Ils expliquent souvent l'impossibilité absolue de mettre en place un tel dispositif. "Je ne suis pas scientifique mais j'ai quand même compris assez rapidement que 460 volts, c'était quand même énorme et donc, qu'il y avait sans doute une arnaque, un montage, quelque chose » (extrait entretien 17)

\section{L'incohérence du contrat de communication}

Les membres du public observent de nombreuses incohérences, aussi bien dans l'habillage du plateau que dans la logique générale de communication. «On peut pas y croire en fait, rien que par rapport aux décors, c'est pas fini, c'est pas cohérent. Dans la boîte, il y a trois boutons, or il y a toujours que 4 possibilités, c'est pas cohérent et puis le branchement c'est pas très cohérent " (extrait entretien 45). Cette affirmation est fausse, il y avait bien quatre boutons dans la cabine, néanmoins, cet individu est persuadé de l'incohérence et elle est structurante dans

57. Weissberg, op.cit.., p. 170. 
l'évaluation qu'il fait de la situation. De très nombreuses personnes commentent le fait que dans une émission de télévision, on ne délaisse jamais l'un des candidats, comme c'est le cas pour Jean-Paul que personne ne vient délivrer à la fin du jeu.

J'ai l'impression que l'émission elle était pas tout à fait préparée. . . le candidat en fait, j'ai eu l'impression qu'on lui avait pas expliqué le jeu, il reposait des questions à l'animatrice et l'animatrice, elle avait pas l'air de lui répondre de façon claire en fait. Donc pour une émission de télévision, j'ai l'impression que c'était pas du direct d'abord et que c'était vraiment bâclé. (extrait entretien 3)

Les membres du public proposent, à plusieurs reprises, des analyses qui révèlent leur culture médiatique. Un jeu télévisé qui serait vraiment fondé sur l'exercice de la torture aurait forcément cherché à exploiter les ressorts du sensationnel et aurait montré des images de souffrance à son auditoire.

Le fait qu'on le voit pas lui (Jean-Paul) ; ça perd en crédibilité. Une émission vraiment sur du voyeurisme, et ce genre de choc aurait fait un gros plan sur sa tête en train de se décomposer parce qu'il prend de l'électricité dans la main. (extrait entretien 17)

L'incohérence est très souvent signalée en ce qui concerne la logique du jeu. Cet argument est souvent un pivot essentiel de l'argumentation des publics : "Comment on peut faire un jeu où même quand on ne répond pas aux questions, on gagne? Quelle est la logique du jeu de faire arriver jusqu'au bout mais sans répondre aux questions, c'est plus un jeu. » (extrait entretien 10)

\section{Le jeu comme cadre interprétatif}

Le fait que les membres du public soient venus assister à un jeu est fondamental. Le jeu et l'institution "jeu télévisé » sont en réalité l'instance suprême de réglage du travail interprétatif. Or comme l'ont expliqué Roger Caillois ou Jacques Henriot, le jeu et l'activité ludique se caractérisent par la définition d'un espace et d'une pratique propre séparés « du reste de l'existence ${ }^{58}$. Henriot définit l'attitude ludique à partir de 3 critères : la distanciation d'avec l'objet pratiqué, la déréalisation, qui engage la duplicité du joueur qui sait qu'il est en train de jouer et enfin l'illusion, qui engage un principe de réalité spécifique ${ }^{59}$.

La formule de "La Zone Xtrême » autorise les membres du public à jouer et ils jouent. Ils sont très nombreux à tenter de répondre aux 27 questions. Le jeu télévisé est un objet connu, il fait l'objet d'une culture personnelle ; les membres du public citent de nombreux jeu, qu'ils regardent ou connaissent. Ils analysent la situation à partir de cette référence, de son efficacité vis-à-vis de ses publics et sa capacité à durer dans une grille de programmes, quand bien même le principe du jeu est jugé désagréable. "Moi je pense que ça ne peut pas être pérenne ! L'émotion elle va venir pendant une émission, deux, trois. . mais pas pendant 50 émissions, parce que ça va être toujours la même chose » (extrait entretien 33).

58. Caillois, R., 1967[1958], Les Jeux et les Hommes. Le Masque et le Vertige, Gallimard, Paris, p. 37.

59. Henriot, J., 1979, Le jeu, PUF, Paris. 


\section{La fiction du gain}

La référence à l'appât du gain, lorsqu'on interroge le public sur les motivations du candidat, est révélatrice de l'emprise de la fiction de jeu. En effet, comme l'analyse très justement un internaute, sur le forum consacré au documentaire, le questionneur est mis en situation de faire comme s'il y avait de l'argent à gagner, même s'il sait qu'il ne gagnera rien. Le joueur doit anticiper les motivations d'un vrai joueur et continuer lorsqu'on l'y exhorte. «Il doit imaginer l'impact qu'aurait cette promesse de gain sur un vrai joueur. À ce moment précis, la promesse de gain n'agit pas sur sa cupidité, comme ce serait le cas s'il s'agissait d'un vrai jeu, mais sur son désir d'être un «bon » sujet d'expérience, désir qu'elle renforce ${ }^{60}$. La majorité des membres du public cite l'appât du gain, alors même que la situation a été élucidée : "Les gens feraient n'importe quoi pour de l'argent » (extraits de plusieurs entretiens). Ce qui est intéressant, c'est que les personnes qui convoquent la question de l'appât du gain ne font pas du tout référence à la soumission à l'autorité, mais au caractère vénal de l'être humain pour justifier le comportement. Le modèle de motivation est pauvre mais il désengage le rapport à la télévision, d'une relation d'obéissance.

\section{Les règles du jeu}

Les membres du public s'attendent donc à un jeu ; Ils définissent le jeu comme étant une forme de réalité particulière. À ce titre, on peut rappeler la définition que Leveneur propose du jeu télévisé, " émission impliquant la participation active d'un ou plusieurs candidats, vedettes ou anonymes, dans des épreuves physiques et/ou intellectuelles et/ou artistiques et dont le déroulement est soumis à des règles contraignantes. Ces règles doivent être porteuses d'un intérêt pour le téléspectateur en lui permettant de participer par délégation, virtuellement ou à distance. L'émission doit se clore par la désignation d'un vainqueur, ayant accompli avec succès toutes les épreuves et/ou n'ayant pas été éliminé. Cette victoire déclarée se traduit par l'obtention d'un gain ou d'un bénéfice, matériel ou symbolique, direct ou indirect ${ }^{61}$. Le public va jusqu'à fantasmer parfois le jeu. Il le dramatise. Au cours de plusieurs entretiens, certains membres du public racontent avoir vu et entendu des choses qu'ils ne peuvent avoir vues et entendues, ce qui est révélateur d'un grand besoin de schéma narratif. « Le point fort de ce jeu, c'est quand il y a les étincelles, c'est impressionnant» (extrait entretien 12).

Par ailleurs, le public sait ce qu'un jeu engage et respecte, à savoir des règles du jeu, des ressorts du divertissement, etc. Le jeu fait l'objet d'une confiance. «Vous auriez été au bout si vous aviez été à la place du questionneur ? Oh oui, j'aurais été jusqu'au bout! Sans problème, de toute façon il ne faut jamais hésiter dans un jeu ! il faut toujours aller jusqu'au bout. » (extrait entretien 23).

À ce titre, deux membres du public partent et expliquent aux membres de l'équipe du plateau que leur déception provient uniquement du fait qu'ils se rendent compte qu'il ne s'agit pas d'un jeu. "Au départ, on vient pour faire

60. TocquevilParis, 21 mas 2010 à $11: 01: 02$.

61. Leveneur, L., 2009, Les travestissements du jeu télévisé, Histoire et analyse d'un genre protéiforme, 1950-2004, Presses Sorbonne nouvelle, p. 30 
une émission, un pilote... [l'emploi transitif du verbe faire est révélateur du programme d'activité de cet interviewé] et là, on fait partie d'une expérience. On se retrouve dans une ambiance "expérience ", à regarder des gens et franchement ça ne m'intéresse pas du tout ! Moi j'aime pas en fait être piégé dans un truc dans lequel on vous dit «c'est une émission de jeu » alors que c'est une expérience, c'est pas la même chose! » (extrait entretien 42).

Par ailleurs, la pauvreté des enjeux du jeu est systématiquement signalée et critiquée. «La Zone Xtrême » ne ressemble guère à un jeu et cela pose problème même lorsque les individus sont en situation de feintise ; la forme d'interaction qu'elle engage avec son public est simple ; Elle est jugée " plat-plat et mou-mou ", " trop monocorde ». Les membres du public s'ennuient. L'animatrice répète inlassablement les mêmes phrases, sur le même ton. Le jeu n'est pas réaliste. Il en va même de cette interrogation : l'expérience est-elle valable si le jeu n'est pas bon?

Ainsi l'hypothèse d'interprétation des publics est avant tout celle d'un jeu, plus qu'une hypothèse de réalité de la torture ; puis ils basculent vers le doute entre l'hypothèse de jeu et l'hypothèse d'expérimentation, ce qui engage une reformulation des questions qu'ils se posent. Ce cadre interprétatif permet de dire que pour les membres du public, les questions évoluent peu à peu. Au début du jeu, on passe de "Est-ce que les décharges peuvent être vraies? » à " Est-ce que le candidat croit que les décharges sont vraies? ». Puis pendant le jeu, on passe de « À quoi je joue? », à « Qui joue avec moi ? »

\section{Interroger sa propre posture de public Le public caution}

On voit que les membres du public analysent leur situation et opèrent un retour réflexif sur leur propre responsabilité en tant qu'acteur d'une situation, dans laquelle les rôles se définissent à la fois mutuellement et à l'avance. Certaines personnes m'expliquent que le public et l'animatrice sont des garanties pour le candidat. Selon eux, le public et l'animatrice cautionneraient les actes de la production et la situation tout entière. Le candidat pense que ce qui est en train de se passer est moral. Le candidat s'autorise à continuer selon eux, car il a ces deux garants autour de lui.

En faisant cela, le public se donne un rôle signifiant. Il fait état d'une intelligence de la situation de communication : il est installé sur le plateau avant que le candidat n'entre, il est donc dans une situation où il préexiste à l'expérience que va faire le candidat. Il est donc certainement mieux informé que lui.

\section{Le public, sujet de l'expérience}

Le public déclare plusieurs fois être "manipulé ». Une fois qu'on lui annonce la feintise, une jeune fille me dit qu'elle est déçue. Ce sentiment d'avoir été dupée alors que tous déclarent que la situation aurait été intolérable si elle avait été réelle et qu'ils savaient que tout était faux, révèle un point intéressant : la difficulté de passer d'une situation de communication à une autre et la confiance avec laquelle ils s'étaient engagés dans le jeu. « Je pense qu'on juge plus le public que le candidat ! pour voir jusqu'où va aller le public ! (extrait entretien 20) 
D'une certaine manière, les membres du public semblent mobiliser une figure $\mathrm{du}$ public qui ne serait plus celle du témoin mais celle du cobaye, ressort de la situation de communication. Ils co-produisent ainsi l'expérience au sens fort. Nombreux sont ceux qui s'interrogent alors sur la valeur sociale de leur comportement : leurs réactions peut-elle être taxée de passivité ? Pourquoi alors n’ont-ils pas cherché à aider le candidat ? Que vont penser leurs proches ? «Si c'est une vraie émission c'est beaucoup plus intéressant je trouve, justement, sur le fait qu'on est restés ici ; après on se pose la question soi-même quand même, en se disant : mince, je me suis pas levée, j'aurais pu me lever et puis voilà quoi... sur le fait d'accepter tout cela, quoi. » (extrait entretien 2).

\section{La révolte du public}

Les cameramen ont toujours posé aux personnes interrogés la même question : «Pourquoi ne pas s'être levé pour dénoncer ce que vous voyiez? ». L'enquête par questionnaire montre qu'avant la révélation de la feintise, $64 \%$ des membres du public déclarent ne pas avoir eu envie d'intervenir pour arrêter le jeu (contre 26,2 \% de « oui »). Après révélation, cette proportion ne baisse que très légèrement.

Les arguments qui sont avancés sont de deux ordres. Certains expliquent que la question n'a pas forcément de sens dans la mesure où ils savaient que l'émission était truquée. $72 \%$ déclarent avant révélation de la feintise qu'ils ont eu le sentiment que les chocs étaient fictifs. Par ailleurs, une grande majorité mobilise une représentation et un respect de la situation médiatique qui révèle une connaissance du travail, et des acteurs à l'œuvre dans ces espaces.

Mais en même temps, je me suis dit, je ne vais pas interrompre l'émission... (extrait entretien 5).

Non, on ne sort pas pendant l'émission, je fais attention à l'équipe qui travaille ici (extrait entretien 42).

Comme l'explique Yves Jeanneret, «dans l'expérience actuelle, [le public] est partie prenante d'une production symbolique destinée à devenir une émission, un message adressé à un public. Les participants ne peuvent pas se poser la question de la responsabilité de la même façon : arrêter l'expérience de Milgram revenait à mettre en difficulté un protocole scientifique. Quitter l'émission, c'est envoyer balader tout le dispositif médiatique, avec ce que cela suppose de travail, d'argent, et de désir conscient ou inconscient d'avoir, un jour, son heure de médiatisation devant un très grand public $»^{62}$.

Les caméras, le «stress des personnes qui travaillent », la disposition organisée de chaque élément engagent un décalage des hypothèses traditionnelles du bien et du mal, du vrai et du faux, etc. "Là les gens sont dans un environnement, ils se disent voila, c'est la télévision, c'est un plateau, je pense que les gens font parfois ce qu'on leur dit de faire. Oui mais là on n'est pas dans la vraie vie, il y a les lumières, il y a la présentatrice qui pousse le candidat à continuer, qui lui

62. Jeanneret, Y., 2010, « $81 \%$ des candidats vont au bout, mais $100 \%$ du public s'abstient d'intervenir ». Interview d'Héléne Marzolf pour Télérama.fr, http://television.telerama.fr/television/ 81-des-candidats-vont-au-bout-mais-100-du-public-s-abstient-d-intervenir,53696.php. 
dit qu'il doit respecter des règles auxquelles il a signé avant de passer... » (extrait entretien 50). La révolte se joue peut-être après, dans les débats que la situation va occasionner entre les membres d'une famille, dans le refus de signer le droit à l'image, dans la participation au forum, et surtout face aux véritables émissions qui utilisent les ressorts de la perversité et de la souffrance, etc.

\section{UNE HYPOTHÈSE DE LA COHÉRENCE}

«Si vous pensiez que c'était du chiqué, si vous ne vouliez pas, dès le début, soit adhérer au concept soit vous laisser entre guillemets berner, vous auriez pu partir? " Cette question posée par l'un des cameramen est révélatrice de la logique avec laquelle est interprété à l'avance le comportement des individus : partir serait le signe de la véritable incrédulité et de la révolte ; rester serait le signe de l'adhésion ou de la crédulité. En posant ce cadre, on refuse la possibilité pour les membres $\mathrm{du}$ public de questionner dans un temps long une logique de communication et de construire un ajustement à un dispositif dont la spécificité est de plonger l'individu à la fois dans le connu (le jeu) et l'inconnu (l'expérimentation). Accepter un postulat (celui du jeu), puis un autre (celui de l'expérimentation), ne revient pas à perdre son sens critique, ni à abdiquer devant une autorité reconnue comme telle. Cela revient plutôt à se donner les conditions d'une compréhension possible des situations. La question que l'on peut se poser est de savoir pourquoi les membres du public engagent à la fois un travail interprétatif intense, régi par le doute, et une sur-ritualisation de leur rôle dès la deuxième séquence de jeu. Ce qui semble apparaître, est que les publics de «La Zone Xtrême », en d'autres termes des publics engagés dans une situation de communication complexe, maintiennent un rapport extrêmement tendu avec le dispositif du plateau de jeu. Pour le comprendre, pour le saisir, il s'y immerge et développe en même temps plusieurs « modes de lecture » pour reprendre la terminologie proposée par Roger Odin : un mode spectacularisant (voir un objet comme un spectacle), fictionnalisant (voir un objet pour vibrer au rythme des événements fictifs racontés) mais aussi documentarisant (voir un objet pour s'informer sur la réalité des choses du monde) lorsqu'il anticipe le public de téléspectateurs à qui sera diffusé le documentaire et peut-être même un mode privé lorsque les cameramen les interrogent (voir un film en faisant retour sur son vécu et/ou sur celui du groupe auquel on appartient). En jouant intensément des rôles, on peut penser que le public fait état d'une prise de distance avec le dispositif en même temps qu'il introduit des espaces de cohérence très temporaires, qu'il abandonnera peut-être plus tard ou renégociera au fur et à mesure de l'expérience.

Cet article a cherché à montrer que le public de la fausse émission de jeu « La Zone Xtrême » construit un ajustement face à une situation médiatique qui convoque des figures de public, des discours et des normes très différentes (du jeu à la torture) ainsi que des régimes de croyances variés. Le public explore le dispositif, il accueille ses différentes redéfinitions, il produit et structure un doute (nourri d'incrédulité et de culture du jeu télévisé), et il éprouve un espace confiné où les corps sont surexposés. Il démontre que la relation aux dispositifs est un ajustement permanent : on peut rester sur un plateau en se demandant ce qu'on y fait, obéir sans y croire, prendre le temps d'interpréter des éléments différents sans 
adhérer à un principe général. Cette analyse va dans le sens de la thèse d'un grand pouvoir de la télévision, mais pas d'un pouvoir totalitaire qui impliquerait une obéissance aveugle. Les publics de « La Zone Xtrême » s'engagent volontiers, dans un rapport conscient de confiance et de défiance, dans un rapport de fascination et de curiosité. Ce temps pris pour l'ajustement au spectacle télévisé est, je l'espère, un jalon supplémentaire dans la construction d'une culture médiatique aiguisée. L'exemple des publics de "La Zone Xtrême » aura permis de montrer que les situations médiatiques engagent des rapports de communication dans lesquels les représentations et les figures fonctionnent comme des éléments clefs dans la construction d'un comportement et d'une interprétation. Ces figures et ces représentations sont mobilisées par des individus qui ont une culture médiatique et qui puisent dedans. L'ajustement des publics au dispositif révèle cette culture et la nourrit.

\section{CAMILLE JUTANT}

